\title{
In Vitro efficacy and antiprotease activity of an antimicrobial ophthalmic drug combination against corneal pathogens of horses
}

\author{
Gil Ben-Shlomo ${ }^{1 *}$, Dennis E. Brooks ${ }^{2}$ and Caryn E. Plummer ${ }^{2}$ \\ *Correspondence: gil@iastate.edu \\ 'Department of Veterinary Clinical Sciences, College of Veterinary Medicine, lowa State University, Ames, IA, USA. \\ 2Department of Small Animal Clinical Sciences, College of Veterinary Medicine, University of Florida, Gainesville, FL, USA.
}

\begin{abstract}
Background: Despite limited information, topical drug combinations are being routinely used by equine veterinarians for the treatment of equine keratitis in order to minimize the intensive labor and costs associated with such treatment with individual drugs. Typical drug combination includes antimicrobials, atropine and antiproteases for the treatment of infection, uveitis and keratomalacia respectively. The goal of this study was to evaluate the in vitro efficacy of an ophthalmic drug combination against protease activity and common equine corneal pathogens.

Methods: Multiple representative isolates of three bacterial and two fungal corneal pathogens of horses were subjected to minimum inhibitory concentration testing of a drug combination that consisted of equal volumes of natamycin, tobramycin, and cefazolin. Proteinase inhibitory activity of the drug combination was assessed by use of a fluorescence microplate assay with gelatin and collagen I as substrates. The Minimal inhibitory concentrations were compared between the drug combination and each of its components; antiproteinase activity of the drug combination was compared with that of serum by use of paired t-tests and a one-way analysis of variance, respectively.

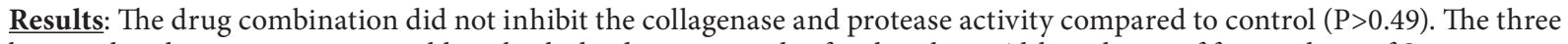
bacterial pathogens were susceptible to both the drug mix and cefazolin alone. Although one of four isolates of $\mathrm{S}$. aureus was resistant to tobramycin alone, the rest of the isolates showed susceptibility to tobramycin. None of the bacterial isolates was sensitive to natamycin alone. All fungal isolates were susceptible to the drug combination and natamycin alone, with the exception of one Fusarium isolate which was susceptible to the drug combination but not to natamycin alone. None of the fungal isolates was susceptible to cefazolin or tobramycin alone.

Conclusions: This drug combination effectively inhibited common corneal pathogens of horses in vitro, but was not effective as an antiprotease treatment. Combining anitimicrobial drugs with serum, does not increase the antimicrobials potency, but reduces the efficacy of the serum as an antiprotease agent.
\end{abstract}

Keywords: Drug combination, antiprotease, equine, tobramycin, natamycin, cefazolin

\section{Introduction}

Ulcerative keratitis is an important and eye threatening disease in horses [1-6]. Infection and tear film hyperprotease activity manifested as "melting cornea" or keratomalacia are important components of corneal ulceration in horses. Horses with severe ulcerative keratitis often need aggressive topical antimicrobial and antiprotease treatment as often as every 1-2 hours for several weeks. The high frequency, single-drug application of topical medications, is expensive and time intensive. Veterinarians are increasingly utilizing drug combinations for the treatment of equine keratitis. Typical drug combinations include antimicrobials, atropine and antiproteases for the treatment of infection, uveitis and keratomalacia respectively. Such combinations can potentially reduce the time and expenses associate with delivery of multiple topical medications.

Scotty et al., [2] showed that an ophthalmic drug combination consisting of equal volumes of natamycin $3.33 \%$, cefazolin $5.5 \%$, tobramycin $0.3 \%$ and equine serum had varied effects in vitro against common equine corneal pathogens and equine tear film proteases. The antimicrobial component of the drug combination inhibited growth of bacteria and fungi, but the level of antiprotease activity that drug combination was found to be less than that of pure equine serum [2].

We hypothesized that the serum alone contributed to the antiprotease activity of the drug combination of Scotty et al., [2]. In addition, we hypothesized that there is no difference between the antimicrobial properties of the drug combination with or without serum. The aim of this study was to evaluate the level of antiprotease activity of a drug combination that contains no serum but only antimicrobial drugs. In addition, the antimicrobial efficacy of the drug combination without the equine serum was tested against most of the same isolates used in previous study [2].

\section{Materials and methods Antimicrobial drugs}

The antimicrobial drugs natamycin, cefazolin and tobramycin were evaluated alone or in combination. The DC was produced 

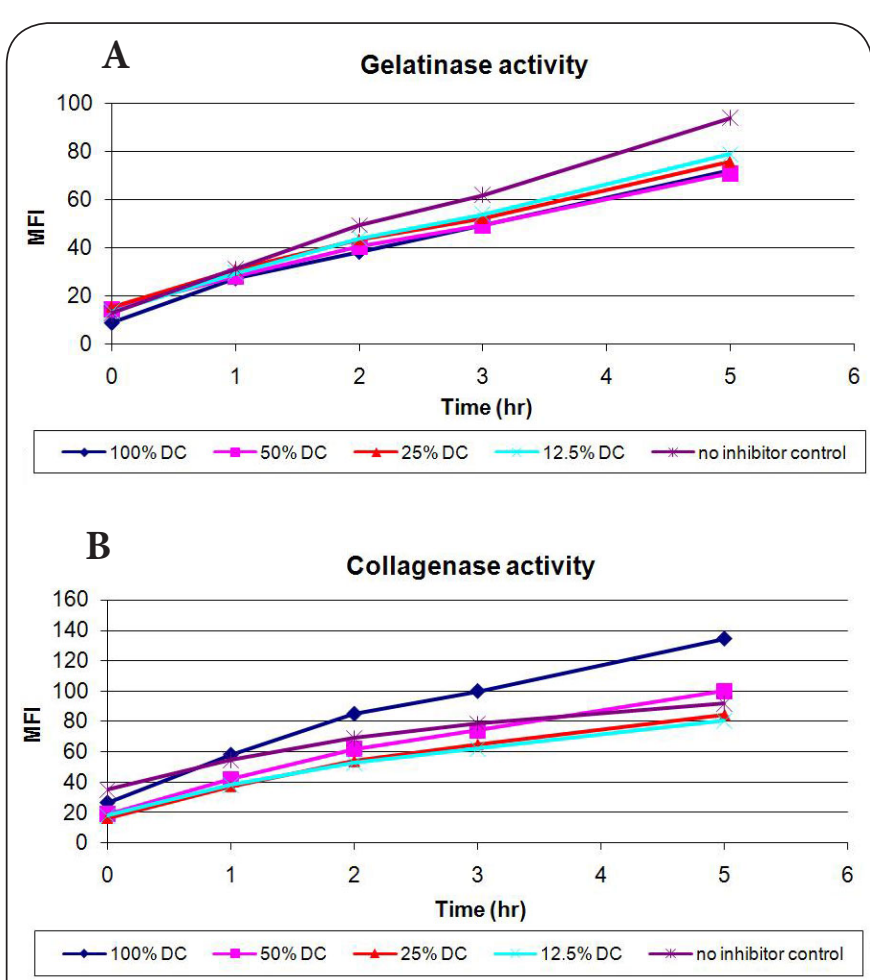

Figure 1. Both gelatinase (A) and collagenase (B) activity of CHP were found to be linear and correlated directly to time over the 5 hours of this study (R2=0.99 and 0.94 , respectively). None of the different DC concentrations inhibited the gelatinase or collagenase activity compared to the positive control ( $\mathrm{P}>0.55$ and $\mathrm{P}>0.3$, respectively).

by mixing equal volumes of natamycin, cefazolin and tobramycin utilizing starting concentrations of $3.33 \%, 5.5 \%$ and $0.3 \%$ respectively. The starting concentration of each drug was the same as we utilize clinically when the drug is used alone.

\section{Antiprotease activity}

Both gelatinase and collagenase inhibitory activity of the DC was quantified by use of a commercially available gelatinasecollagenase assay ${ }^{\mathrm{a}}$ conducted in accordance with manufacturer protocols. Briefly, solutions of fluorescein-quenched gelatin and collagen I were placed in microplate wells. Then, CHP which has both collagenase and gelatinase activity, was then added to these wells at $0.2 \mathrm{U} / \mathrm{mL}$ for the gelatin assays (to induce gelatinase activity) and $1.0 \mathrm{U} / \mathrm{mL}$ for the collagen assays (to induce collagenase activity) Four different DC concentrations $(100 \%, 50 \%, 25 \%$ and $12.5 \%)$ were added to the microplate wells in duplicates in order to test their antiprotease activity. The various concentrations were prepared via dilution with the reaction buffer provided in the assay kit immediately prior to incubation. Destruction of proteins, with consequent release of fluorescein molecules, was quantified by measuring the MFI at $\mathrm{t}=0,1,2,3$ and 5 hours post incubation in room temperature using a microplate reader. Reaction buffer (instead of DC) was used as a positive control (no protease inhibition), and 1,10-phenanthroline was used as a negative control (protease inhibition) sample. Mean \pm SD fluorescein excitation and emission were set at $485 \pm 20$ and $528 \pm 20 \mathrm{~nm}$, respectively. All components were added to the wells at the same time, $\mathrm{t}=0$. The antiprotease activity was compared to the positive control after subtracting the background MFI. Background MFI was measured from wells containing the DC (in the appropriate concentration), the substrate (gelatin or collagen), and buffer instead of CHP. The negative control served as a background of the positive control. Each DC concentration test was performed in duplicates. All wells content were re-suspended (due to precipitation of the natamycin) by pipet, right before each reading of the plate.

\section{Effect on antimicrobial growth}

Common equine bacterial corneal pathogens were identified by searching the University of Florida Veterinary Medical Center database from 2000 through 2005 [2]. Three bacterial species and two fungal species were subjected to in vitro MIC testing of the three different antimicrobial drugs in accordance with a standard protocol $[7,8]$.

The drug combination had been prepared $\leq 24$ hours before MIC testing and stored at $4^{\circ} \mathrm{C}$ until used. Each MIC test for this study was performed in triplicate by use of custom designed plates ${ }^{b}$ and the dilution of the DC that successfully inhibited microbial growth was determined.

\section{Statistical analyses}

The antiprotease activity of the DC was compared with that of the negative control and the positive control by use of paired t- test. Values of $P<0.05$ were considered statistically significant.

\section{Results \\ Antiprotease activity}

Both gelatinase and collagenase activity of $\mathrm{CHP}$ were found to be linear and correlated directly to time over the 5 hours of this study ( $R^{2}=0.99$ and 0.94 , respectively). None of the different DC concentrations inhibited the gelatinase or collagenase activity compared to the positive control $(\mathrm{P}>0.55$ and $\mathrm{P}>0.3$, respectively) (Figure 1 ).

\section{Antimicrobial efficacy}

The database search yielded 266 corneal bacterial isolates and 28 corneal fungal isolates as detailed by Scotty et al., [2] . Among the most common bacteria, beta-hemolytic Staphylococcus spp (7 isolates), a-hemolytic Streptococcus spp (3 isolates) and Staphylococcus aureus (4 isolates) were chosen due to availability of the original isolates. Aspergillus and Fusarium spp were the 2 most common fungal isolates. Four of the original Aspergillus isolates and and 6 of the original Fusarium isolates were available for this study.

Growth of the three bacterial pathogens was inhibited by both the drug combination mixture (dilution $<0.00006 \mathrm{X}$ ) 
Ben-Shlomo et al. Veterinary Medicine and Animal Sciences 2013, http://www.hoajonline.com/journals/pdf/2054-3425-1-2.pdf

Table 1. Bacterial isolates: MIC $(\mu \mathrm{g} / \mu \mathrm{L})$ of the individual antimicrobial drugs and the dilution of DC of which bacterial growth was inhibited is presented for each isolate. Asterisk $\left(^{*}\right)$ indicates susceptibility.

\begin{tabular}{|c|c|c|c|c|c|}
\hline \multirow{2}{*}{ Organism } & \multirow{2}{*}{ Isolate } & \multirow{2}{*}{$\begin{array}{c}\mathrm{DC} \\
\text { (Dilution) }\end{array}$} & \multicolumn{3}{|c|}{$\mathrm{MIC} \mu \mathrm{g} / \mu \mathrm{L}$} \\
\hline & & & Tobramycin & Cefazolin & Natamycin \\
\hline$\beta$ staph & 1 & $<0.00006 \mathrm{X}^{*}$ & $<0.25^{*}$ & $<1^{*}$ & 256 \\
\hline$\beta$ staph & 2 & $<0.00006 \mathrm{X}^{\star}$ & $<0.25^{\star}$ & $<1^{\star}$ & 256 \\
\hline$\beta$ staph & 3 & $<0.00006 \mathrm{X}^{*}$ & $<0.25^{*}$ & $2^{*}$ & 256 \\
\hline$\beta$ staph & 4 & $<0.00006 \mathrm{X}^{*}$ & $<0.25^{*}$ & $<1^{*}$ & 256 \\
\hline$\beta$ staph & 5 & $<0.00006 \mathrm{X}^{*}$ & $<0.25^{*}$ & $<1^{*}$ & 256 \\
\hline$\beta$ staph & 6 & $<0.00006 \mathrm{X}^{\star}$ & $<0.25^{\star}$ & $<1^{\star}$ & $>128$ \\
\hline$\beta$ staph & 7 & $<0.00006 \mathrm{X}^{\star}$ & $2^{*}$ & $4^{*}$ & $\geq 512$ \\
\hline a strep & 1 & $<0.00006 \mathrm{X}^{\star}$ & $8^{*}$ & $<1^{\star}$ & 128 \\
\hline a strep & 2 & $<0.00006 \mathrm{X}^{\star}$ & $0.5^{*}$ & $<1^{*}$ & 256 \\
\hline a strep & 3 & $<0.00006 \mathrm{X}^{*}$ & $0.5^{*}$ & $<1^{*}$ & 512 \\
\hline S. aureus & 1 & $<0.00006 \mathrm{X}^{\star}$ & $0.5^{*}$ & $<1^{\star}$ & $>512$ \\
\hline S. aureus & 2 & $<0.00006 \mathrm{X}^{\star}$ & $<0.25^{*}$ & $<1^{\star}$ & $>512$ \\
\hline S. aureus & 3 & $<0.00006 \mathrm{X}^{\star}$ & $<0.25^{\star}$ & $<1^{\star}$ & $>512$ \\
\hline S. aureus & 4 & $<0.00006 \mathrm{X}^{*}$ & 16 & $2^{*}$ & $>512$ \\
\hline
\end{tabular}

Table 2. Fungal isolates: MIC $(\mu \mathrm{g} / \mu \mathrm{L})$ of the individual antimicrobial drugs and the dilution of DC of which fungal growth was inhibited is presented for each isolate. Asterisk $\left(^{*}\right)$ indicates susceptibility.

\begin{tabular}{lcc|ccc}
\hline \multirow{2}{*}{ Organism } & \multirow{2}{*}{ Isolate } & \multirow{2}{*}{$\begin{array}{c}\text { DC } \\
\text { (Dilution) }\end{array}$} & \multicolumn{3}{|c}{ MIC $\mu \mathrm{g} / \mu \mathrm{L}$} \\
\cline { 5 - 6 } & & & Tobramycin & Cefazolin & Natamycin \\
\hline Aspergillus & 1 & $0.004 \mathrm{X}^{*}$ & $>128$ & $>512$ & $32^{*}$ \\
Aspergillus & 2 & $0.001 \mathrm{X}^{*}$ & $>128$ & $>512$ & $8^{*}$ \\
Aspergillus & 3 & $0.004 \mathrm{X}^{*}$ & $>128$ & $>512$ & $32^{*}$ \\
Aspergillus & 4 & $0.004 \mathrm{X}^{*}$ & $>128$ & $>512$ & $32^{*}$ \\
Fusarium & 1 & $0.001 \mathrm{X}^{*}$ & $>128$ & $>512$ & $8^{*}$ \\
Fusarium & 2 & $0.001 \mathrm{X}^{*}$ & $>128$ & $>512$ & $4^{*}$ \\
Fusarium & 3 & $0.004 \mathrm{X}^{*}$ & $>128$ & $>512$ & $>512$ \\
Fusarium & 4 & $0.001 \mathrm{X}^{*}$ & $>128$ & $>512$ & $8^{*}$ \\
Fusarium & 5 & $0.001 \mathrm{X}^{*}$ & $>128$ & $>512$ & $4^{*}$ \\
Fusarium & 6 & $0.001 \mathrm{X}^{*}$ & $>128$ & $>512$ & $8^{*}$ \\
\hline
\end{tabular}

and cefazolin alone, but not to natamycin alone. While one of four isolates of $S$. aureus was resistant to tobramycin alone, growth of the other three isolates was inhibited by it (Table 1). Growth of both Aspergillus and Fusarium was inhibited by both the DC (dilution $\leq 0.004 \mathrm{X}$ ) and the natamycin alone, with the exception of one Fusarium isolate which was resistant to natamycin but not to the DC (Table 2).

\section{Discussion}

This study shows that a drug combination consisting of equal volumes of natamycin $3.33 \%$, cefazolin $5.5 \%$ and tobramycin $0.3 \%$ effectively inhibits growth of common bacteria and fungus isolated from horses' cornea, in vitro. The absence of serum from the DC in our study did not diminish this effect compared to DC used in the study of Scotty et al., [2]. This was expected since the antibacterial drugs in our combination were diluted less than in the DC reported by Scotty et al., (3X vs. 4X). In the current study, the DC as well as natamycin alone were effective against the fungal isolates. Scotty et al., [2] showed that the similar DC and serum mixture was significantly more effective against fungal isolates than natamycin alone. That can possibly be explained by the properties of serum as antimicrobial agent $[\mathbf{9 , 1 0}$. However, it is important to remember that the MIC of the different components of the DC was back calculated by Scotty et al., [2]: the MIC of each component medication was calculated by multiplying the MIC of the drug combination by 0.25 since each component comprised $25 \%$ of the drug combination. This method does not consider drug interaction (and possible drug synergy) into account as a conflicting factor; hence it probably does not represent a true MIC. Moreover, any statistics used to compare the DC MIC to its individual component is extremely problematic for the same reason. Therefore, we are suggesting the DC and its individual components be compared in terms of the susceptibility (reflected by inhibition of growth) or resistance of the examined microorganism.

Both gelatinase and collagenase activity of $\mathrm{CHP}$ were found to be linear and correlated directly to time over the 5 hours of this study ( $R^{2}=0.99$ and 0.94 , respectively), in agreement with Scotty et al., [2]. None of the different DC concentrations inhibited the gelatinase or collagenase activity compared to the positive control ( $P>0.55$ and $P>0.3$, respectively) (Figure 1 ). It can be concluded that the antiprotease activity of the drug combination in Scotty's et al., [2] report is contributed by the equine serum alone. A drug combination with serum demonstrated reduced antiprotease activity compared to the pure serum. This reduced antiproteases activity is probably due to the dilution of serum (1:4) within the drug combination, raising concerns regarding the benefit of serum in drug combinations. In our view, serum should not be diluted for clinical use but should be administered by itself to achieve a full antiprotease effect.

Veterinarians often apply drug mixtures via automated pumps set for administration of drugs for 24 hour periods (personal communication). A major concern when administering ophthalmic suspensions such natamycin via pumps is that they may result in precipitation of the active ingredient. During our study we found that the DC in our study needed vigorous pipetation after 1 hour of incubation in order to resuspend the natamycin. Using the automated pumps without refrigeration can further reduce the efficacy of drugs that need refrigeration such as natamycin, cefazolin and serum.

Drug combinations have the potential of minimizing the time, stress, and fatigue associated with topical treatment regimens consisting of multiple drugs used separately for horses with keratitis. Nevertheless factors such as refrigeration 
and sedimentation (if used in an automated pump) as well as drug interaction, $\mathrm{pH}$ and long term stability may affect the practicality of its use. Further research, including in vivo studies, is necessary in order to learn the efficacy and properties of drug combinations prior to utilizing them in the clinic.

\section{List of abbreviations}

CHP: Clostridium histolyticum proteinase

DC: Drug combination

MIC: Minimal inhibitory concentration

MFI: Mean fluorescence intensity

\section{Competing interests}

The authors declare that they have no competing interests.

\section{Authors' contributions}

\begin{tabular}{|l|c|c|c|}
\hline Authors' contributions & GBN & DEB & CEP \\
\hline Research concept and design & $\checkmark$ & $\checkmark$ & -- \\
\hline Collection and/or assembly of data & $\checkmark$ & -- & -- \\
\hline Data analysis and interpretation & $\checkmark$ & $\checkmark$ & -- \\
\hline Writing the article & $\checkmark$ & -- & -- \\
\hline Critical revision of the article & -- & $\checkmark$ & $\checkmark$ \\
\hline Final approval of article & $\checkmark$ & $\checkmark$ & $\checkmark$ \\
\hline Statistical analysis & $\checkmark$ & -- & -- \\
\hline
\end{tabular}

\section{Acknowledgement}

The authors would like to thank the American Quarter Horse Foundation for their support of this study.

Publication history

Editor: Cengiz Gokbulut, Balikesir University, Turkey.

EIC: Olivier A. E. Sparagano, Northumbria University, UK.

Received: 26-Sep-2013 Revised: 08-Oct-2013

Accepted: 17-Oct-2013 Published: 15-Nov-2013

\section{References}

1. Brooks DE and Matthews AG. Equine ophthalmology. In: Gelatt KN, ed. (4th ed), Veterinary Ophthalmology. 2007; 1165-1274.

2. Scotty NC, Brooks DE and Schuman Rose CD. In vitro efficacy of an ophthalmic drug combination against corneal pathogens of horses. Am J Vet Res. 2008; 69:101-7. | Article | PubMed

3. Brooks DE, Plummer CE, Kallberg ME, Barrie KP, Ollivier FJ, Hendrix DV, Baker A, Scotty NC, Utter ME, Blackwood SE, Nunnery CM, Ben-Shlomo G and Gelatt KN. Corneal transplantation for inflammatory keratopathies in the horse: visual outcome in 206 cases (1993-2007). Vet Ophthalmol. 2008; 11:123-33. | Article | PubMed

4. de Linde Henriksen M, Plummer CE, Mangan B, Ben-Shlomo G, Tsujita $\mathrm{H}$, Greenberg S, Toft $\mathrm{N}$ and Brooks DE. Visual outcome after corneal transplantation for corneal perforation and iris prolapse in $\mathbf{3 7}$ horses: 1998-2010. Equine Vet J. 2012; 44 Suppl 43:115-9. | Article I PubMed

5. Brooks DE, Plummer CE, Mangan BG and Ben-Shlomo G. Equine subepithelial keratomycosis. Vet Ophthalmol. 2013; 16:93-6. | Article | PubMed

6. Williams LB and Pinard CL. Corneal ulcers in horses. Compend Contin Educ Vet. 2013; 35:E4. | Article I PubMed

7. National Committee for Clinical Laboratory Standards. Reference method for broth dilution antifungal susceptibility testing of filamentous fungi. Approved standard. M38-A2. Wayne, PA: National Committee for Clinical Laboratory Standards, 2008. | Website

8. Lorian V. Antibiotics in Laboratory Medicine 5th Edition. 2005.
9. Bezkorovainy A. Antimicrobial properties of iron-binding proteins. Adv Exp Med Biol. 1981; 135:139-54. | Article | PubMed

10. Dahl MV. Dermatophytosis and the immune response. J Am Acad Dermatol. 1994; 31:S34-41. | Article | PubMed

\section{Citation:}

Ben-Shlomo G, Brooks DE and Plummer CE. In Vitro efficacy and antiprotease activity of an antimicrobial ophthalmic drug combination against corneal pathogens of horses. Vet Med Anim Sci. 2013; 1:2.

http://dx.doi.org/10.7243/2054-3425-1-2 